\title{
Virulência de isolados de Colletotrichum gloeosporioides de populações selvagens de Stylosanthes spp.
}

\author{
Eduardo Alano Vieira ${ }^{(1)}$, Maria José d’Avila Charchar(1), Marilia Santos Silva ${ }^{(1)}$ \\ e José Ribamar Nazareno dos Anjos ${ }^{(1)}$
}

\begin{abstract}
(1)Embrapa Cerrados, BR 020, Km 18, Caixa Postal 08223, CEP 73310-970 Planaltina, DF. E-mail: vieiraea@cpac.embrapa.br, mdavila@cpac.embrapa.br, marilia@cpac.embrapa.br, ribamar@cpac.embrapa.br
\end{abstract}

\begin{abstract}
Resumo - Os objetivos deste trabalho foram determinar o padrão de virulência/avirulência e as raças de 274 isolados brasileiros de Colletotrichum gloeosporioides de populações selvagens de Stylosanthes spp. dos estados de Goiás, Bahia e Minas Gerais, em 12 acessos diferenciadores, e agrupar os isolados de acordo com a similaridade em virulência. Culturas monospóricas dos isolados foram aspergidas sobre plântulas de 12 acessos diferenciadores. A aferição dos resultados (virulência/avirulência) foi realizada dez dias após as inoculações. A maioria dos isolados brasileiros de $C$. gloeosporioides de populações selvagens de Stylosanthes spp. apresenta baixa capacidade de virulência, e a raça predominante não apresenta virulência a nenhum dos acessos diferenciadores avaliados. Não existe padrão de distribuição da variabilidade em virulência entre os isolados em função do estado de origem.
\end{abstract}

Termos para indexação: antracnose, raças fisiológicas, série diferencial, leguminosa forrageira tropical, resistência genética.

\section{Virulence of Colletotrichum gloeosporioides isolates from wild Stylosanthes spp. populations}

\begin{abstract}
The objectives of this work were to determine the virulence/avirulence pattern and the races of 274 isolates of Colletotrichum gloeosporioides from wild populations of Stylosantes spp. from the states of Goiás, Bahia and Minas Gerais, in 12 differential plants as well as to group the isolates according to of their similarity in virulence. Monosporic cultures of the isolates were inoculated onto 12 accessions of differential plants. Results (virulence/avirulence) were surveyed ten days after inoculation. Most Brazilian isolates of C. gloeosporioides from wild populations of Stylosanthes spp. presented low virulence capacity, the predominant race does not present virulence to any of the accessions of differential plants evaluated and besides there is no pattern of virulence variability distribution among the isolates by state of origin.
\end{abstract}

Index terms: anthracnose, physiological races, differential series, tropical legume forage, genetic resistance.

\section{Introdução}

Determinadas espécies forrageiras do gênero Stylosanthes destacam-se entre as leguminosas tropicais por sua elevada capacidade produtiva, rusticidade, qualidade nutricional, adaptação a diferentes condições climáticas, tolerância à seca, tolerância ao alumínio, tolerância a pastejo pesado e capacidade de recuperação de solos degradados, especialmente quanto à fixação de nitrogênio (Gardener, 1984; Andrade et al., 2004). O principal centro de origem e diversidade do gênero é o Brasil, porém o gênero pode ser encontrado naturalmente desde a América Central até a América do Sul (Willians et al., 1984).
A antracnose, causada pelo fungo Colletotrichum gloeosporioides (Penz.) Penz. \& Sac. fase teleomórfica Glomerella cingulata (Stonem.) Spauld. \& Schrenk., é a moléstia mais prejudicial e prevalente do gênero Stylosanthes. Ocorre em todas as regiões onde a forrageira é cultivada, e é mais severa na época das chuvas, uma vez que o fungo necessita de umidade relativa do ar acima de $95 \%$ e de uma camada de água livre na folha para iniciar a infecção. Entretanto, elevadas precipitações reduzem sua severidade (Davis, 1987).

A utilização de Stylosanthes de forma comercial vêm sendo limitada, principalmente em razão da susceptibilidade da maioria das cultivares à antracnose (Milles \& Lascano, 1997), aliada à baixa produtividade 
de sementes (Andrade et al., 2004). Essa moléstia causa prejuízos à cultura, uma vez que ataca toda a parte aérea da planta, afeta a produtividade (forragem e sementes), o estabelecimento e a persistência no campo de cultivares susceptíveis (Chakraborty, 2004). Diante disso, cresce a importância da utilização de cultivares geneticamente resistentes. Entretanto, a resistência tem se mostrado transiente (Davis et al., 1984; Trutmann, 1994; Cameron et al., 1997; Guodao et al., 1997; Miles \& Lascano, 1997).

A elevada plasticidade genética do fungo é apontada como a principal causa para a rápida seleção de raças virulentas às cultivares lançadas como resistentes, o que dificulta o controle da moléstia (Davis et al., 1984; Miles \& Lascano, 1997). Portanto, há necessidade de estudos sobre a variabilidade em virulência das populações do patógeno, em especial no Brasil, considerado o centro de origem do patossistema (Chakraborty et al., 2004a).

Pesquisas realizadas com isolados coletados em populações selvagens do hospedeiro na América do Sul mostram a existência de ampla variabilidade em virulência nas populações do patógeno (Kelemu et al., 1996; Chakraborty et al., 2002, 2004a; Charchar et al., 2003) e que a variabilidade em virulência das populações sul-americanas é superior à das populações da Austrália, Índia e China (Chakraborty et al., 1996, 2004b).

Os objetivos deste trabalho foram determinar o padrão de virulência/avirulência e as raças de 274 isolados brasileiros de Colletotrichum gloeosporioides de populações selvagens de Stylosanthes spp. dos estados de Goiás, Bahia e Minas Gerais, em 12 acessos diferenciadores e agrupar os isolados de acordo com a similaridade em virulência.

\section{Material e Métodos}

No período de 1997 a 2000, foram coletadas folhas, caules e hastes com sintomas de antracnose (C. gloeosporioides), em populações selvagens de Stylosanthes spp. em 24 municípios dos Estados de Goiás, Minas Gerais e Bahia (Tabela 1), com distância de aproximadamente $50 \mathrm{~km}$ entre cada ponto de coleta. As amostras coletadas foram acondicionadas, isoladamente, em envelopes de papel com a identificação do local e data de coleta.

No Laboratório de Fitopatologia da Embrapa Cerrados, em Planaltina, DF, pedaços de aproximadamente $5 \mathrm{~mm}$ das amostras coletadas de tecido infectado foram desinfestadas em $1 \%$ de $\mathrm{NaOCl}$, durante
2 min, e lavadas com água estéril. Depois da secagem em papel estéril, o material foi transferido para meio de cultura batata-dextrose-ágar (BDA) com estreptomicina e incubado a $26^{\circ} \mathrm{C}$ por uma semana. A cultura monospórica de cada isolado, obtida a partir de um único esporo germinado, foi preservada em pequenos frascos com água estéril e em tubos com meio de cultura (BDA) à temperatura constante de $20^{\circ} \mathrm{C}$.

Os isolados foram multiplicados em meio ágar-aveia, em câmara de crescimento a $26^{\circ} \mathrm{C}$, com fotoperíodo de 12 horas. Após dez dias, a suspensão de esporos foi filtrada em gaze dupla esterilizada, para remoção do micélio do patógeno e ajustada para a concentração de $10^{6}$ conídios por mL. Cerca de 35 dias após a semeadura, a suspensão de esporos foi aspergida em plântulas dos 12 acessos diferenciadores de Stylosanthes spp. (Tabela 2). Foram utilizados os 11 acessos diferenciadores indicados por Chakraborty et al. (2002) e mais a cultivar Mineirão, adicionada à série diferencial por sua relevância no Brasil (Charchar et al., 2003; Andrade et al., 2004). Depois desse procedimento, as plântulas foram incubadas em câmara úmida por 48 horas a $26^{\circ} \mathrm{C}$.

Tabela 1. Relação dos 274 isolados de Colletotrichum gloeosporioides utilizados com indicação do número de isolados dos municípios e estados de coleta.

\begin{tabular}{lcc}
\hline Município & Estado & $\mathrm{N}^{0}$ de isolados \\
\hline Alvorada do Norte & GO & 3 \\
Curvelo & MG & 5 \\
Bom Despacho & MG & 2 \\
Bom Jesus da Lapa & BA & 4 \\
Buritis & MG & 31 \\
Cabeceiras & GO & 33 \\
Caetité & BA & 18 \\
Brumado & BA & 2 \\
Capão da Volta & BA & 6 \\
Correntina & BA & 4 \\
Diamantina & MG & 71 \\
Janaúba & MG & 1 \\
Barreiras & BA & 3 \\
João Pinheiro & MG & 2 \\
Lençóis & BA & 33 \\
Luislândia & MG & 29 \\
Padre Bernardo & GO & 1 \\
Paracatu & MG & 2 \\
Pirapora & MG & 10 \\
Posse & GO & 1 \\
Roda Velha & BA & 3 \\
São João da Ponte & MG & 2 \\
Três Marias & MG & 2 \\
Urucuia & MG & 6 \\
\hline Total & & 274 \\
\hline & &
\end{tabular}


Dez dias após a aspersão dos acessos diferenciadores, foi efetuada a aferição dos resultados, por meio da escala de notas descrita por Chakraborty (1990) e adaptada para a avaliação de isolados brasileiros do fungo por Charchar et al. (2003), que varia de 0 a 9 , em função da porcentagem de tecido necrosado, em que 0 , sem sintomas; 1,1 a $3 \% ; 2,4$ a $6 \% ; 3,7$ a $12 \%$; 4,13 a $25 \%$; 5,26 a $50 \% ; 6,51$ a $75 \% ; 7,76$ a $87 \% ; 8,88$ a $94 \%$ e 9,95 a $100 \%$. As notas de 0 a 3 foram consideradas como indicativo de resistência do hospedeiro (infecção baixa/avirulência) e as notas de 4 a 9 foram consideradas como indicativo de suscetibilidade do hospedeiro (infecção alta/virulência), segundo o critério proposto por Charchar et al. (2003). Dessa forma, cada isolado apresentou padrão próprio de tipos de infecção alta e baixa (virulência/avirulência) quanto aos acessos diferenciadores utilizados.

Em decorrência das dificuldades oriundas do estabelecimento de critérios para a determinação do grau de agressividade do patógeno, a melhor maneira para a determinação das raças é por meio da análise multivariada, considerando a relativa agressividade dos isolados e, assim, os agrupando pela similaridade em agressividade Chakraborty (2004). Entretanto, essa estratégia pode levar a problemas quando outros pesquisadores tentam comparar os resultados obtidos em seus trabalhos com os obtidos por outros grupos de pesquisa. Neste trabalho é proposto uma alternativa que também se baseia no nível de agressividade do patógeno, para a determinação da raça de isolados do patógeno. Para estabelecer essa alternativa, foi selecionado um conjunto de acessos diferenciadores robusto e utilizado um critério definido para a distinção entre alto e baixo nível de infecção e, a partir dessas decisões, foram determinadas as raças dos patógenos.

Os isolados receberam denominações de acordo com o sistema de nomenclatura proposto neste trabalho (Tabela 2), que utiliza o mesmo princípio do sistema norte-americano de nomenclatura da ferrugem-da-folha-da-aveia (Puccinia coronata f. sp. avenae), desenvolvido por Chong et al. (2000). Os 12 acessos diferenciadores foram dispostos em três subconjuntos de quatro diferenciadores; para cada subconjunto, existem apenas 16 combinações de tipos infecção alta e baixa, arranjados em um sistema binário. Foi utilizado um código composto de 16 letras consoantes entre B e T para identificar cada uma das 16 combinações de tipos infecção alta e baixa (virulência/avirulência) (Tabela 2). O código resultante, composto por três letras,

Tabela 2. Arranjo dos subconjuntos de quatro acessos diferenciadores de Stylosanthes spp. utilizados na determinação das raças de Colletotrichum gloesporioides.

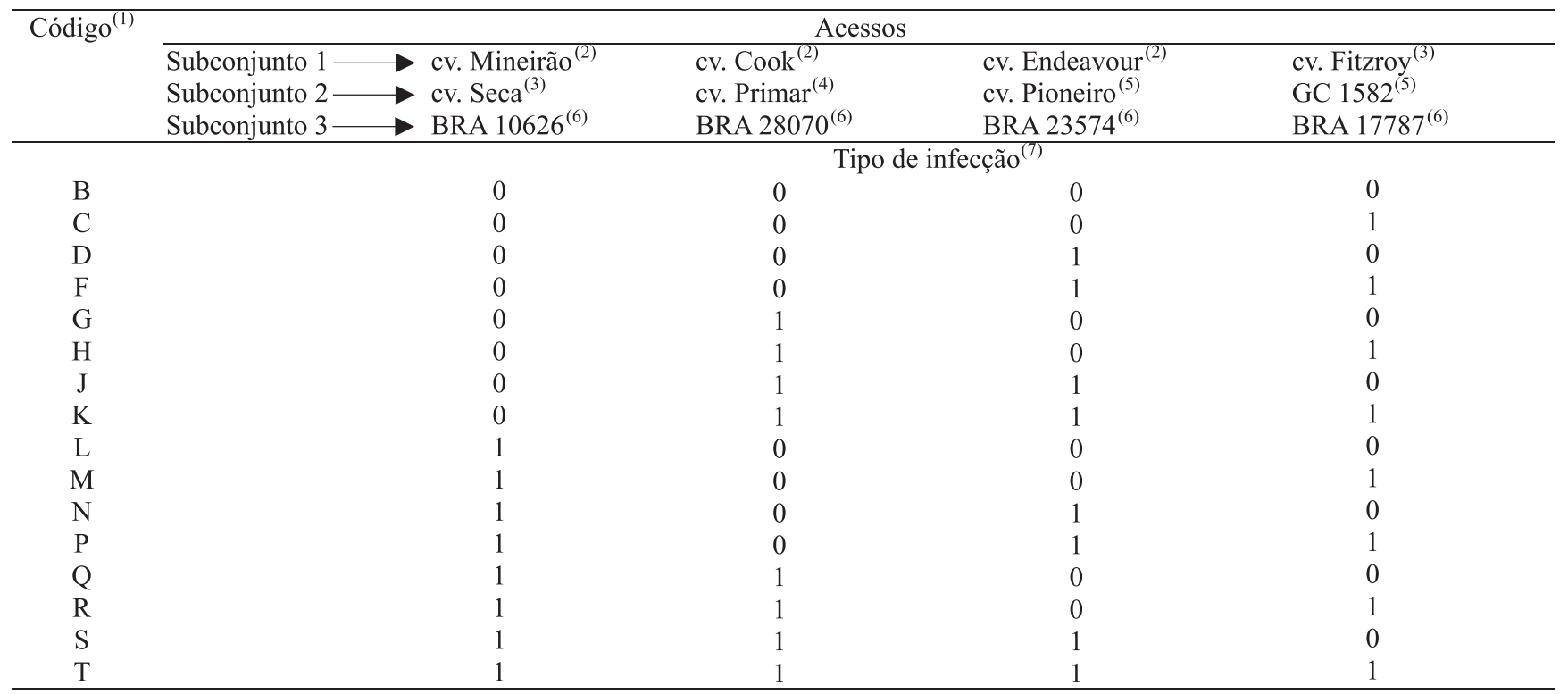

(1)Código: é a designação do subconjunto 1 seguida pela do subconjunto 2 e pela do subconjunto 3; por exemplo, a raça $\mathrm{SBP}=$ subconjunto 1 (S), avirulenta somente para Fitzroy; subconjunto 2 (B), avirulenta para todos os acessos; subconjunto 3 (P), avirulenta somente para BRA 028070. ${ }^{(2)}$ Stylosanthes guianensis. ${ }^{(3)}$ Stylosanthes scabra. ${ }^{(4)}$ Stylosanthes seabrana. ${ }^{(5)}$ Stylosanthes macrocephata; $\mathrm{CG}=$ número do acesso na Embrapa Gado de Corte. ${ }^{(6)}$ Stylosanthes capitata, em que: BRA 10626 = GC 1084; BRA 28070 = GC 1081; BRA $23574=$ GC $1094 ;$ BRA $17787=$ GC 1086. ${ }^{(7)} 0=$ tipo infecção baixa (reação de avirulência); 1 = tipo infecção alta (reação de virulência). 
foi utilizado para a designação das raças do patógeno. Esse sistema permite o acréscimo de novos subconjuntos de quatro acessos diferenciadores e assim a inclusão de letras adicionais à direita das utilizadas no sistema.

Os dados dos padrões de virulência/avirulência dos isolados para cada um dos acessos diferenciadores foram transformados para uma matriz de dados binários em que 1, infecção alta/virulência e 0 , tipo infecção baixa/ avirulência. Esta matriz foi utilizada na estimativa da similaridade em virulência entre cada um dos pares de isolados por meio do índice de coincidência simples (ICS), conforme a equação: $\mathrm{ICS}=\mathrm{C} / \mathrm{N}$, em que $\mathrm{C}$ é o número de acessos diferenciadores com o mesmo padrão de virulência ou avirulência e $\mathrm{N}$ é o número total de acessos diferenciadores avaliados.

A similaridade em virulência foi transformada em dissimilaridade segundo a equação: $D_{i j}=1-S_{i j}$, em que $\mathrm{D}_{\mathrm{ij}}$ é a dissimilaridade em virulência entre cada par de $\mathrm{i}$ e j isolados; $S_{\mathrm{ij}}$ é a similaridade em virulência entre cada par de $\mathrm{i}$ e $\mathrm{j}$ isolados. Com base na matriz de dissimilaridade, os isolados foram agrupados pelo método de agrupamento de Tocher para obter homogeneidade dentro dos grupos e heterogeneidade entre grupos. Todas as análises estatísticas foram realizadas com o programa Genes (Cruz, 2001).

\section{Resultados e Discussão}

A aspersão dos 274 isolados nos 12 acessos diferenciadores de Stylosanthes spp. resultou em um total de 3.288 inoculações, das quais 328 (10\%) revelaram reação de virulência ou infecção alta, e 2.960
(90\%), reação de avirulência ou infecção baixa, o que evidenciou baixa capacidade de virulência da maioria dos isolados (Tabela 3), com predomínio de notas zero, ou imunidade, entre todas as reações aferidas. Resultado semelhante foi relatado por Chakraborty et al. (2002) que, ao avaliar 296 isolados coletados no Brasil, observaram reduzido número de reações de virulência, com a inoculação dos 296 isolados sobre 11 dos acessos diferenciadores utilizados neste trabalho, excetuandose a cultivar Mineirão.

Entre os acessos diferenciadores utilizados, nenhum foi resistente a todos os isolados ou revelou completa susceptibilidade (Tabela 3). Entretanto, Chakraborty et al. (2002), ao avaliar 296 isolados brasileiros do fungo, não relataram a ocorrência de isolados virulentos à cultivar Primar, que neste trabalho apresentou a menor frequiência de isolados virulentos (3\%). O acesso diferenciador com maior freqüência de isolados virulentos foi o Fitzroy (28\%) e a freqüência de isolados virulentos aos demais acessos foi intermediária, de 5 a 14\% (Tabela 3). Resultados semelhantes foram relatados por Chakraborty et al. (2002), que reportaram a ocorrência de muitos isolados virulentos à cultivar Fitzroy.

Com base nos 12 acessos diferenciadores de Stylosantes spp., foi possível classificar os 274 isolados em 62 raças distintas (Tabela 4), por meio do sistema de nomenclatura proposto (Tabela 2). A raça predominante foi a BBB que ocorreu 132 vezes. Essa raça não expressou capacidade de virulência a nenhum dos diferenciadores e foi detectada nos três estados em que foram coletados os isolados (Tabela 4). A segunda raça mais freqüente

Tabela 3. Reação de virulência dos 274 isolados de Colletotrichum gloeosporioides avaliados sobre 12 acessos diferenciadores de Stylosanthes spp.

\begin{tabular}{|c|c|c|c|c|}
\hline Acesso diferenciador & Espécie & País de origem & Ano de lançamento & $\mathrm{N}^{\mathrm{o}}$ de isolados virulentos (\%) \\
\hline cv. Mineirão & Stylosanthes guianensis & Brasil & 1993 & $25(9 \%)$ \\
\hline cv. Cook & Stylosanthes guianensis & Austrália & 1971 & $35(13 \%)$ \\
\hline cv. Endeavour & Stylosanthes guianensis & Austrália & 1971 & $37(14 \%)$ \\
\hline cv. Fitzroy & Stylosanthes scabra & Austrália & 1979 & $78(28 \%)$ \\
\hline cv. Seca & Stylosanthes scabra & Austrália & 1976 & $38(14 \%)$ \\
\hline cv. Primar & Stylosanthes seabrana & Austrália & 1996 & $7(3 \%)$ \\
\hline cv. Pioneiro & Stylosanthes macrocephala & Brasil & 1983 & $16(6 \%)$ \\
\hline GC 1582 & Stylosanthes macrocephala & Brasil & $\mathrm{NLC}^{(1)}$ & $14(5 \%)$ \\
\hline BRA 10626 & Stylosanthes capitata & Brasil & NLC & $29(11 \%)$ \\
\hline BRA 28070 & Stylosanthes capitata & Brasil & NLC & $16(6 \%)$ \\
\hline BRA 23574 & Stylosanthes capitata & Brasil & NLC & $17(6 \%)$ \\
\hline BRA 17787 & Stylosanthes capitata & Brasil & NLC & $16(6 \%)$ \\
\hline Total & & & & $328^{(2)}(10 \%)$ \\
\hline
\end{tabular}

${ }^{(1)}$ Não lançado comercialmente. ${ }^{(2)}$ Número total de reações de virulência (\%). 
foi a CBB, que ocorreu 25 vezes e apresentou virulência a apenas um dos acessos diferenciadores, e foi detectada nos três estados em que foram coletados os isolados (Tabela 4).

Os isolados estudados foram coletados em populações selvagens de Stylosanthes spp., as quais não sofreram seleção artificial para resistência à antracnose, e, por isso, espera-se que os genótipos das populações apresentem individualmente poucos genes verticais de resistência ao fungo, em virtude de não terem sido submetidos a um processo de acúmulo de genes de resistência. Dessa forma, isolados portadores de muitos genes de virulência conteriam genes desnecessários e, assim, expressariam uma desvantagem competitiva em relação aos que não os apresentam (Vanderplank, 1963). Conseqüentemente, isolados com genes desnecessários à virulência tenderiam a ocorrer em menor freqüência.

A hipótese de que o processo de seleção natural levou à manutenção de raças de $C$. gloeosporioides com poucos genes de virulência nas populações brasileiras selvagens de Stylosanthes spp. é plausível. Portanto, raças com poucos genes de virulência dominam essas populações, em razão da não difusão de genótipos melhorados de Stylosanthes spp. com genes de resistência raça-específica. Não existem razões teórico-biológicas, para o surgimento de raças complexas de patógenos em populações selvagens de hospedeiros com elevada variabilidade genética. Entretanto, Chakraborty et al. (2002) e Weeds et al. (2003) relataram a ocorrência de raças mais complexas em áreas próximas a estações de pesquisa. Esta situação provavelmente foi decorrente da

Tabela 4. Relação das 62 raças de Colletotrichum gloeosporioides identificadas, com a indicação da sua nomenclatura (raça), do número e porcentagem de isolados pertencentes a cada raça e do número de acessos entre os 12 diferenciadores a que cada raça foi virulenta (№ V) e avirulenta (№ Av).

\begin{tabular}{|c|c|c|c|c|c|c|c|c|c|}
\hline Raça ${ }^{(1)}$ & $\mathrm{N}^{\mathrm{o}}$ de isolados $(\%)$ & $\mathrm{N}^{\mathrm{o}} \mathrm{V}$ & $\mathrm{N}^{\mathrm{o}} \mathrm{Av}$ & Estado & Raça & $\mathrm{N}^{\mathrm{o}}$ de isolados $(\%)$ & $\mathrm{N}^{\mathrm{o}} \mathrm{V}$ & $\mathrm{N}^{\mathrm{o}} \mathrm{Av}$ & Estado \\
\hline$\overline{\mathrm{BBB}}$ & $132(48,18)$ & 0 & 12 & GO; MG e BA & CLT & $1(0,36)$ & 6 & 6 & MG \\
\hline CBB & $25(9,12)$ & 1 & 11 & $\mathrm{GO} ; \mathrm{MG}$ e $\mathrm{BA}$ & CLB & $12(4,38)$ & 2 & 10 & BA e MG \\
\hline BBS & $1(0,36)$ & 3 & 9 & MG & DDB & $1(0,36)$ & 2 & 10 & MG \\
\hline $\mathrm{CCS}$ & $1(0,36)$ & 5 & 7 & MG & BDB & $3(1,09)$ & 1 & 11 & $\mathrm{BA}$ e $\mathrm{MG}$ \\
\hline CBM & $1(0,36)$ & 3 & 9 & MG & TBB & $1(0,36)$ & 4 & 8 & MG \\
\hline BBL & $3(1,09)$ & 1 & 11 & MG & TLB & $2(0,73)$ & 5 & 7 & MG \\
\hline $\mathrm{HBQ}$ & $1(0,36)$ & 4 & 8 & MG & MBB & $1(0,36)$ & 2 & 10 & MG \\
\hline $\mathrm{CBL}$ & $2(0,73)$ & 2 & 10 & MG & RBB & $1(0,36)$ & 3 & 9 & MG \\
\hline CBQ & $1(0,36)$ & 3 & 9 & MG & CLC & $1(0,36)$ & 3 & 9 & MG \\
\hline FLN & $1(0,36)$ & 5 & 7 & MG & CMJ & $1(0,36)$ & 5 & 7 & MG \\
\hline $\mathrm{CBJ}$ & $2(0,73)$ & 3 & 9 & MG & FST & $1(0,36)$ & 9 & 3 & MG \\
\hline FCL & $1(0,36)$ & 4 & 8 & MG & CBT & $1(0,36)$ & 5 & 7 & MG \\
\hline DBL & $1(0,36)$ & 2 & 10 & MG & LRB & $1(0,36)$ & 4 & 8 & MG \\
\hline CDT & $1(0,36)$ & 6 & 6 & MG & BLL & $1(0,36)$ & 2 & 10 & MG \\
\hline FBQ & $1(0,36)$ & 4 & 8 & MG & SDB & $2(0,73)$ & 4 & 8 & MG \\
\hline HDD & $1(0,36)$ & 4 & 8 & MG & JLB & $1(0,36)$ & 3 & 9 & MG \\
\hline FBB & $4(1,46)$ & 2 & 10 & MG e GO & TSQ & $1(0,36)$ & 9 & 3 & MG \\
\hline DBB & $8(2,92)$ & 1 & 11 & GO; MG e BA & QBB & $2(0,73)$ & 2 & 10 & MG \\
\hline $\mathrm{BCB}$ & $1(0,36)$ & 1 & 11 & GO & CBD & $1(0,36)$ & 2 & 10 & MG \\
\hline $\mathrm{CCB}$ & $2(0,73)$ & 2 & 10 & GO & GDB & $1(0,36)$ & 2 & 10 & MG \\
\hline JBB & $4(1,46)$ & 2 & 10 & MG e GO & SQL & $1(0,36)$ & 6 & 6 & MG \\
\hline GBB & $7(2,55)$ & 1 & 11 & $\mathrm{BA}$ e $\mathrm{MG}$ & BLB & $5(1,82)$ & 1 & 11 & MG \\
\hline SBM & $2(0,73)$ & 5 & 7 & MG & $\mathrm{BBG}$ & $1(0,36)$ & 1 & 11 & MG \\
\hline SBB & $2(0,73)$ & 3 & 9 & MG & CLG & $1(0,36)$ & 3 & 9 & MG \\
\hline JCF & $1(0,36)$ & 5 & 7 & MG & CLN & $1(0,36)$ & 4 & 8 & MG \\
\hline QFP & $1(0,36)$ & 7 & 5 & MG & CGB & $2(0,73)$ & 2 & 10 & BA e MG \\
\hline GFC & $1(0,36)$ & 4 & 8 & MG & FLB & $1(0,36)$ & 3 & 9 & $\mathrm{BA}$ \\
\hline GFK & $1(0,36)$ & 6 & 6 & MG & CQB & $1(0,36)$ & 3 & 9 & BA \\
\hline SBF & $1(0,36)$ & 5 & 7 & MG & BFB & $2(0,73)$ & 2 & 10 & BA \\
\hline LBB & $7(2,55)$ & 1 & 11 & $\mathrm{BA}$ e $\mathrm{MG}$ & $\mathrm{BBC}$ & $1(0,36)$ & 1 & 11 & BA \\
\hline CLL & $4(1,46)$ & 3 & 9 & MG & GCB & $1(0,36)$ & 2 & 10 & BA \\
\hline
\end{tabular}

(1)Nomenclatura segundo o sistema proposto neste trabalho (Tabela 2). 
elevada variabilidade genética do germoplasma avaliado nesses locais ao longo dos anos (Weeds et al., 2003; Chakraborty et al., 2004a).

As raças que evidenciaram virulência ao maior número de acessos diferenciadores foram FST, TSQ e QFP, com virulência a 9, 9 e 7 acessos, respectivamente (Tabela 4). Cada uma dessas raças foi detectada apenas uma vez, o que corrobora a hipótese de que elas apresentam desvantagem competitiva em relação às raças menos complexas. Quanto à baixa frequência de raças mais complexas, com muitos genes de virulência, o mesmo resultado foi descrito por Chakraborty et al. (2002). De acordo com esses autores raças mais complexas tenderam a não se agrupar com as demais, o que também foi observado neste trabalho (Tabela 5).

A análise de agrupamento de Tocher, em função da dissimilaridade em virulência estimada por meio do padrão de virulência/avirulência das raças aos 12 acessos diferenciadores de Stylosanthes spp., dividiu as 62 raças de C. gloeosporioides identificadas neste trabalho em 13 grupos distintos (Tabela 5). O maior grupo foi o G1, que reuniu 24 raças e 226 isolados dos estados de Minas Gerais, Bahia e Goiás, o que evidencia não existir padrão definido de distribuição da variabilidade em virulência entre os três estados de coleta de isolados (Tabelas 4 e 5), e demonstra que os isolados não apresentaram tendência de agrupamento de acordo com o local em que foram coletados, como relatado por Chakraborty et al. (2002). Os demais grupos reuniram de oito raças (G2 e G3) a uma raça (G10, G11, G12 e G13).

Populações de fungos que ocorrem naturalmente em populações selvagens de Stylosanthes no Brasil, apesar de expressar elevada variabilidade genética (Kelemu et al., 1999; Weeds et al., 2003) e de apresentar variabilidade em virulência superior à das populações da Austrália, Índia e China (Chakraborty et al., 1996, 2004b), não são, na maioria, constituídas por raças portadoras de muitos genes de virulência individualmente. Porém, as raças são portadoras de grande plasticidade genética, em especial, em razão de o fungo apresentar reprodução sexuada nas condições de campo no Brasil (recombinação genética). Assim, é possível inferir que a estratégia de melhoramento para resistência ao fungo, que deve a ser priorizada no Brasil, é a que privilegia a resistência parcial, que normalmente é quantitativa, não é raça específica e se caracteriza por um progresso lento da doença no campo. Deste modo, possivelmente seria evitada a seleção de raças complexas, como acontece, por exemplo, no Brasil, em espécies como aveia (Vieira et al., 2006) e trigo (Chaves \& Barcellos, 2002) em relação à ferrugemda-folha, cujo melhoramento visando à resistência do tipo raça específica ocasionou a seleção de raças do fungo muito complexas, por causa da grande pressão de seleção exercida sobre as populações do patógeno.

Tabela 5. Agrupamento das 62 raças de Colletotrichum gloeosporioides identificadas por meio do método de agrupamento de Tocher, de acordo com a distância genética estimada pelo complemento do índice de coincidência simples, obtido com base no padrão de virulência/avirulência das raças a 12 acessos diferenciadores de Stylosanthes spp., e indicação do número de raças e de isolados de cada grupo de similaridade.

\begin{tabular}{|c|c|c|c|}
\hline Grupo & Raças $^{(1)}$ & № de raças & $\mathrm{N}^{0}$ de isolados \\
\hline$\overline{\mathrm{G} 1}$ & $\begin{array}{l}\text { BBB, CBB, BBL, CBL, CBM, CBQ, CLL, CLB, BLL, BLB, DBL, FBB, DBB, } \\
\text { FLB, CCB, MBB, CBD, CGB, BCB, LBB, BBG, BBC, GBB e BDB }\end{array}$ & 24 & 226 \\
\hline G2 & FLN, CLN, CLT, CBT, BBS, CCS, CBJ e CDT & 8 & 9 \\
\hline G3 & JBB, SBB, TBB, SDB, QBB, RBB, TLB e JLB & 8 & 15 \\
\hline G4 & HBQ, FBQ e FCL & 3 & 3 \\
\hline G5 & HDD, GDB, GFC, BFB e GCB & 5 & 6 \\
\hline G6 & SBM, SBF e JCF & 3 & 4 \\
\hline G7 & CLC, CLG e CQB & 3 & 3 \\
\hline G8 & QFP e GFK & 2 & 2 \\
\hline G9 & TSQ e SQL & 2 & 2 \\
\hline G10 & FST & 1 & 1 \\
\hline G11 & LRB & 1 & 1 \\
\hline G12 & DDB & 1 & 1 \\
\hline G13 & $\mathrm{CMJ}$ & 1 & 1 \\
\hline
\end{tabular}

${ }^{(1)}$ Nomenclatura da raça segundo o sistema proposto neste trabalho (Tabela 2). 


\section{Conclusões}

1. A maioria dos isolados brasileiros de Colletotrichum gloeosporioides de populações selvagens de Stylosanthes spp. apresenta baixa capacidade de virulência.

2. A raça predominante de Colletotrichum gloeosporioides, em populações selvagens de Stylosanthes spp. nos estados de Minas Gerais, Bahia e Goiás, não apresenta virulência a nenhum dos 12 acessos diferenciadores avaliados.

3. Não existe padrão definido de distribuição da variabilidade em virulência entre os isolados de Colletotrichum gloeosporioides das populações selvagens de Stylosanthes spp. dos estados de Minas Gerais, Bahia e Goiás.

\section{Referências}

ANDRADE, R.P.; KARIA, C.T.; RAMOS, A.K.B. Stylosanthes as a forage legume at its centre of diversity. In: CHAKRABORTY, S. (Ed.). High-yielding antracnose-resistant Stylosanthes for agricultural systems. Canberra: Aciar, 2004. p.39-50.

CAMERON, D.F.; TREVORROW, R.M.; LIU, C.J. Recent advances in studies of antracnose of Stylosanthes. II. Approaches to breeding for anthracnose resistance in Stylosanthes in Australia. Tropical Grasslands, v.31, p.424-429, 1997.

CHAKRABORTY, S. Anthracnose disease of Stylosanthes. In: CHAKRABORTY, S. (Ed.). High-yielding anthracnose-resistant Stylosanthes for agricultural systems. Canberra: Aciar, 2004. p.113-124. CHAKRABORTY, S.; FERNANDES, C.D.; CHARCHAR, M.J.; THOMAS, M.R. Pathogenic variation in Colletotrichum gloeosporioides infecting Stylosanthes spp. in a center of diversity in Brazil. Plant Disease, v.92, p.553-562, 2002.

CHAKRABORTY, S.; FERNANDES, C.D.; CHARCHAR, M.J.; WEEDS, P.L.; KELEMU, S. Colletotrichum gloeosporioides diversity at centres of origin in Brazil and Colombia. In: CHAKRABORTY, S. (Ed.). High-yielding anthracnose-resistant Stylosanthes for agricultural systems. Canberra: Aciar, 2004a. p.165-172.

CHAKRABORTY, S.; RAMESH, C.R.; KEXIAN, Y. Colletotrichum gloeosporioides diversity at centres of utilization in Australia, China and India. In: CHAKRABORTY, S. (Ed.). High-yielding anthracnose-resistant Stylosanthes for agricultural systems. Canberra: Aciar, 2004b. p.173-178.

CHAKRABORTY, S.; RATCLIFF, D.; MACKAY, F.J. Anthracnose of Stylosanthes scabra: effect of leaf surface wetness on disease severety. Plant Disease, v.74, p.379-384, 1990.

CHAKRABORTY, S.; THOMAS, M.R.; ELLIS, N. A multivariate analysis of pathogenic variation in Colletotrichum gloeosporioides infecting tropical pasture legume, Stylosanthes scabra. Phytopathology, v.86, p.283-289, 1996.

CHARCHAR, M.J.; ANJOS, J.R.N.; GOMES, A.C.; CHAKRABORTY, S.; FERNANDES, C.D.; AKIMOTO, A.K.; TOMAZ, L.V. Variabilidade patogênica de Colletotrichum gloeosporioides oriundos de populações nativas de Stylosanthes spp. de três estados brasileiros. Planaltina: Embrapa Cerrados, 2003. 18p.
CHAVES, M.S.; BARCELLOS, A.L. Especiação fisiológica de Puccinia triticina no Brasil em 2002. Fitopatologia Brasileira, v.31, p.57-62, 2002.

CHONG, J.; LEONARD, K.J.; SALMERON, J.J. A north american system of nomenclature for Puccinia coronata f. sp. avenae. Plant Disease, v.84, p.580-585, 2000.

CRUZ, C.D. Programa Genes: aplicativo computacional em genética e estatística. Viçosa: UFV, 2001. 648p.

DAVIS, R.D. Seedborne Colletotrichum gloeosporioides infection and fungicidal control in Stylosanthes spp. Seed Science and Technology, v.15, p.785-791, 1987.

DAVIS, R.D.; IRWIN, J.A.G.; CAMERON, D.F. Variation on virulence and pathogenic specialization of Colletotrichum gloeosporioides isolates from Stylosanthes scabra cvs. Fitzroy and Seca. Australian Journal of Agricultural Research, v.35, p.653662, 1984.

GARDENER, C.J. The dynamics of Stylosanthes pasture. In: STACE, H.M.; EDYE, L.A. (Ed.). The biology and agronomy of Stylosanthes. Centercourt: Academic Press, 1984. p.333-357.

GOUDAO, L.; PHAIKAEW, C.; STUR, W.W. Status of Stylosanthes development in other countries. II. Stylosanthes development and utilization in China and south-east Asia. Tropical Grasslands, v.31, p.460-467, 1997.

KELEMU, S.; BADEL, J.L.; MORENO, C.X. Virulence spectrum of South American isolates of Colletotrichum gloeosporioides on selected Stylosanthes guianensis genotypes. Plant Disease, v.80, p.1355-1358, 1996.

KELEMU, S.; SKINNER, D.Z.; BADEL, J.L.; MORENO, C.X.; RODRIGUES, M.X.; FERNANDES, C.D.; CHARCHAR, M.J.; CHAKRABORTY, S. Genetic diversity in South American Colletotrichum gloeosporioides isolates from Stylosanthes guianensis, a tropical forage legume. European Journal of Plant Pathology, v.105, p.261-272, 1999.

MILES, J.W.; LASCANO, C.E. Status of Stylosanthes development in other countries. I. Stylosanthes development and utilization in South America. Tropical Grasslands, v.31, p.454-459, 1997.

TRUTMANN, P. Diseases of tropical pasture plants in Central and South America. In: LENNÉ, J.M.; TRUTMANN, P. (Ed.). Diseases of tropical pasture plants. Wallingford: CAB International, 1994. p.21-42.

VANDERPLANK, J.E. Plant diseases: epidemics and control. New York: Academic Press, 1963. 349p.

VIEIRA, E.A.; CARVALHO, F.I.F.; CHAVES, M.S.; OLIVEIRA, A.C.; BERTAN, I.; MARTINS, A.F.; HARTWIG, I.; BENIN, G.; VALÉRIO, I.V.; FONSECA, D.A.R. Padrão de resistência de genótipos de aveia à ferrugem da folha na definição de hibridações. Pesquisa Agropecuária Brasileira, v.41, p.607-614, 2006.

WEEDS, P.L.; CHAKRABORTY, S.; FERNANDES, C.D.; CHARCHAR, M.J.D.; RAMESH, C.R.; KEXIAN, Y.; KELEMU, S. Genetyc diversity in Colletotrichum gloeosporioides from Stylosanthes spp. at centers of origin and utilization. Phytopathology, v.93, p.176-185, 2003.

WILLIANS, R.J.; RAID, R.; SCHULTZE-KRAFT, R.; SOUZA COSTA, N.M.; THOMAS, B.D. Natural distribution of Stylosanthes. In: STACE, H.M.; EDYE, L.A. (Ed.). The biology and agronomy of Stylosanthes. Sydney: Academic Press, 1984. p.73-101. 